\title{
345 - 7 Critical factors for safely supporting dementia patients in mental health crisis in community
}

Dr Sabarigirivasan Muthukrishnan, Dr Kate Hydon, Lucy Young and Cristie Howells, UK

\section{Background:}

Best practice in dementia care is support in the home. Yet, crisis is common and can result in hospital admission. Home-treatment of crisis is an alternative to hospital admission that can have better outcomes and is the preference of people living with dementia.

\section{Purpose:}

To report an investigation of the management of crisis for people with dementia living at home and managed by a Home Treatment Crisis Team.

\section{Objective:}

To identify critical factors for successful resolution of crisis and avoidance of hospital admission

\section{Methods:}

The research was mixed-methods case study design. It was an in depth investigation of what happens during crisis in people with dementia and how it is managed by a home treatment crisis team to resolution and outcome at six weeks and six months. Methods were observation of the management of crisis in the home setting for 15 people with dementia (max 3 per person, total 41 observations), interviews with people with dementia $(n=5)$, carers $(n=13)$, and 14 professionals (range 1 to 6 per person, total 29), a focus group with professionals $(n=9)$ and extraction from medical records of demographics and medical history.

The analysis focused on the identification of key treatments, behaviours, education and context important for home treatment to prevent hospital admission.

\section{Findings:}

The study recruited 15 of the 88 accepted referrals to the service for management of a crisis in a person with dementia.

The seven key factors key for crisis resolution were

- a systems approach with embedded respect for personhood,

- attention to carer needs independently of the person with dementia,

- review and monitoring of the effect of medications,

- awareness and promotion of potential benefits with treatment at home,

- education of the health and social care workforce in dementia care,

- local availability of respite and other social care services.

- a dynamic and flexible working ethos and meaningful MDT working with flattened hierarchy

The Home Treatment Crisis Team created a 'Safe Dementia Space' for the person with dementia in crisis. In the first instance, this was immediate but temporary with on-going assessment and intervention until negotiated permanent support was in place coproduced and agreed by stakeholders to be a sustainable dementia space with acceptable risk of harm to the person with dementia or others. The approach enabled avoidance of hospital admission in more than $80 \%$ of referrals.

\section{Conclusion:}

This is the first study to collect data during crisis at home for people with dementia and to investigate process and management. It reveals the Home Treatment Crisis Team created sustainable 'Safe Dementia Space' to enable the person with dementia to continue to live in the community during and 
after crisis, thus avoiding hospital admission. The identified key components of the management approach for crisis resolution are important considerations in the design and delivery of home treatment services for people with dementia in the UK and beyond. 